\title{
The Effects of Dilute Sulfuric Acid on Sheet Resistance and Transmittance in Poly(3,4-thylenedioxythiophene): Poly(styrenesulfonate) Films
}

\author{
Teen-Hang Meen, ${ }^{1}$ Kan-Lin Chen, ${ }^{2}$ Yu-Hao Chen, ${ }^{1}$ Wen-Ray Chen, ${ }^{1}$ Dei-Wei Chou, \\ Wen-How Lan, ${ }^{4}$ and Chien-Jung Huang ${ }^{5}$ \\ ${ }^{1}$ Department of Electronic Engineering, National Formosa University, Hu-Wei, Yunlin 63201, Taiwan \\ ${ }^{2}$ Department of Electronic Engineering, Fortune Institute of Technology, Kaohsiung 83160, Taiwan \\ ${ }^{3}$ Department of Aviation \& Communication Electronics, Air Force Institute of Technology, Kaohsiung 82044, Taiwan \\ ${ }^{4}$ Department of Electnical Engineering, National University of Kaohsiung, Kaohsiung 81148, Taiwan \\ ${ }^{5}$ Department of Applied Physics, National University of Kaohsiung, Kaohsiung 81148, Taiwan
}

Correspondence should be addressed to Chien-Jung Huang; chien@nuk.edu.tw

Received 15 September 2013; Accepted 3 October 2013

Academic Editor: Liang-Wen Ji

Copyright (C) 2013 Teen-Hang Meen et al. This is an open access article distributed under the Creative Commons Attribution License, which permits unrestricted use, distribution, and reproduction in any medium, provided the original work is properly cited.

\begin{abstract}
The conductivity of poly(3,4-thylenedioxythiophene): poly(styrenesulfonate) (PEDOT: PSS) films by adding various molar concentrations of sulfuric acid $\left(\mathrm{H}_{2} \mathrm{SO}_{4}\right)$ was improved and studied in this paper. The sheet resistance of the doped PEDOT: PSS film was enhanced with increasing the ratio of $\mathrm{H}_{2} \mathrm{SO}_{4}$, but it drops after the maximum sheet resistance. The reason for this phenomenon is resulting from the fact that the $\mathrm{H}_{2} \mathrm{SO}_{4}$ preferentially react with the sorbitol which is so-called the pinacol rearrangement. The nonconductive anions of some $\mathrm{PSS}^{-}$were substituted by the conductive anions of hydrogen sulfate $\left(\mathrm{HSO}_{4}{ }^{-}\right)$when the residual $\mathrm{H}_{2} \mathrm{SO}_{4}$ reacted with PSS. In addition to the substitution reaction, PEDOT chains were increasingly aggregated with increasing the ratio of $\mathrm{H}_{2} \mathrm{SO}_{4}$. After doped $\mathrm{H}_{2} \mathrm{SO}_{4}$, the sheet resistance of $\mathrm{H}_{2} \mathrm{SO}_{4}$-doped PEDOT: PSS film is improved nearly $36 \%$; the surface roughness is reduced from $1.268 \mathrm{~nm}$ to $0.822 \mathrm{~nm}$ and the transmittance is up to $91.9 \%$ in the visible wavelength range from 400 to $700 \mathrm{~nm}$.
\end{abstract}

\section{Introduction}

The poly(3,4-thylenedioxythiophene): poly(styrenesulfonate) (PEDOT: PSS) is a conductive polymer blend, which mainly consisted of two substances, PEDOT and PSS. The PEDOT is a polymer of the 3,4-thylenedioxythiophene (EDOT), which is insoluble in water. The solubility of the PEDOT increased through being combined with the PSS. Therefore, the PEDOT: PSS becomes easier to use.

The PEDOT: PSS was widely applicated to electronic devices such as organic solar cells, actuators, capacitors, organic light-emitting devices, and sensors [1-3]. One of the fundamental requirements for operation of all organic optoelectronic devices is a stable anode interface. Indium tin oxide (ITO) has been widely used for organic optoelectronic devices due to its high optical transparency and high conductivity. However, the work function of ITO is low. The most common way is to be coated with a buffer layer on the top of ITO surface. The buffer layer can export the carriers more efficiently.

Among these buffer layers, the PEDOT: PSS is superior to other materials due to its structural stability, optical transparency, and process ability. The PEDOT: PSS as the buffer layer not only increases the work function of the ITO but also planarizes the surface of ITO substrate. The insufficient conductivity of the PEDOT: PSS is a restriction for application in optoelectronic devices, although the PEDOT: PSS has the above advantages. Therefore, the topic of enhancement on conductivity of the PEDOT: PSS was studied by many researchers. The research of the PEDOT: PSS 
indicates that the conductivity of the PEDOT: PSS film can be increased by the addition of organic solvents, such as sorbitol [4-7], dimethyl sulfoxide (DMSO) [7-10], glycerol [7, 11-13], ethylene glycol (EG) [14-16], or polyethylene glycol (PEG) [17].

In this paper, the conductivity of PEDOT: PSS solutions was improved through adding the inorganic solution, dilute sulfuric acid. The PEDOT: PSS solutions were prepared with different molar concentrations of dilute sulfuric acid to induce variations on the surface morphology and the electrical properties of PEDOT: PSS films. The mechanism of enhancement conductivity for PEDOT: PSS films was further studied through measuring instruments. These experimental results provided further evidences for our proposed mechanism.

\section{Experimental}

The SIGMA D-Sorbitol (98\%) doped the Clevios PH 500 PEDOT: PSS was used as the solution for preparation of thin films by the spin-coating method. The sorbitol was added to the PEDOT: PSS solution directly, and then the doped PEDOT: PSS solution was stirred for $30 \mathrm{~min}$ at room temperature. The mixed solution was doped again by adding different molar concentrations of dilute sulfuric acid. At last, the mixed PEDOT: PSS solution is the so-called double doped PEDOT: PSS solution. Glass substrates with an area of $2 \times 2 \mathrm{~cm}^{2}$ were precleaned with acetone, methanol and deionized (DI) water in an ultrasonic bath for 10 min each time, sequentially. Finally, glass substrates were dried with nitrogen. The double doped PEDOT: PSS solution was coated by spinner on the cleanly glass substrate and formed the double doped PEDOT: PSS film. The spin-coating was performed at a rotation rate of $3500 \mathrm{rpm}$ for $20 \mathrm{sec}$. The double doped PEDOT: PSS film was heated at $150^{\circ} \mathrm{C}$ for $20 \mathrm{~min}$ on a hotplate in ambient lab conditions.

The sheet resistance of double doped PEDOT: PSS films was measured with four point sheet resistivity meter (SRM103, Solar Energy Tech., Taiwan). The surface morphology and roughness of double doped PEDOT: PSS films were measured by atomic force microscopy (AFM, Park Systems, XE-70). And the transmittance of double doped PEDOT: PSS films were measured by UV/visible spectrometer (HITACHI, U-3900).

\section{Results and Discussion}

The electrical property of the PEDOT: PSS is strongly dependent on its chemical and physical structures. The chemical structures of the PEDOT and the PSS are shown in Figure 1. The $-\mathrm{OH}$ groups of the PSS structure were dissociated to $\mathrm{H}^{+}$in the water. And the $\mathrm{H}^{+}$actively attacked the double bond of the thiophene of the PEDOT to form hydrogen bonding. However, electrons of the $\mathrm{C}-\mathrm{O}$ shared electron pairs were attracted by $\mathrm{O}$ due to its higher electronegativity. The phenomenon led to the fact that the $\mathrm{C}$ of the $\mathrm{C}-\mathrm{O}$ bond became a positive charge. Finally, the ionic bond was formed between the $\mathrm{C}^{+}$of the PEDOT structure and the $\mathrm{O}^{-}$of the PSS<smiles>CC(C)CC(CC(CC(CC(CC(C)c1ccc(S(=O)(=O)O)cc1)c1ccc(S(=O)(=O)O)cc1)c1ccc(S(=O)(=O)O)cc1)c1ccc(S(=O)(=O)O)cc1)c1ccc(S(=O)(=O)O)cc1</smiles>

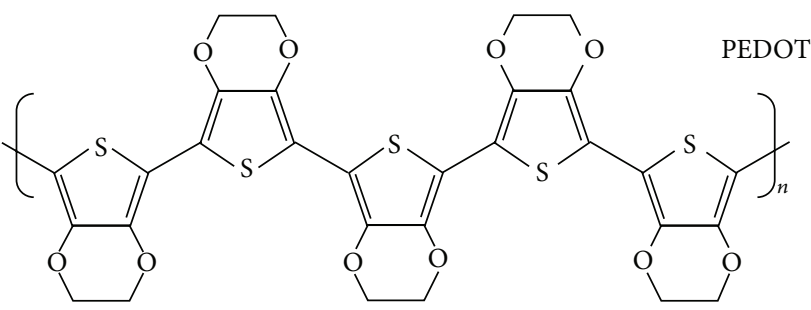

FIGURE 1: Chemical structure of PEDOT: PSS.

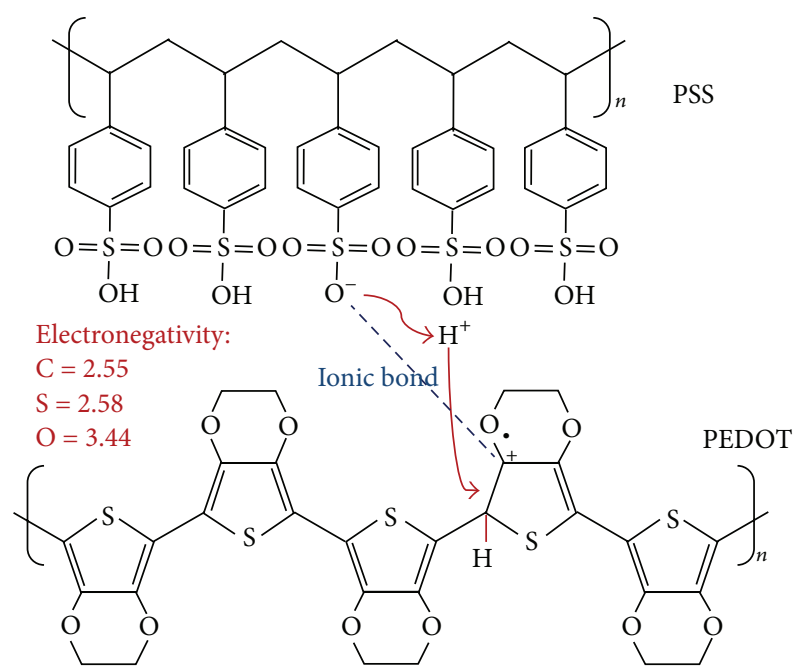

FIGURE 2: Schematic illustration of the reaction between PEDOT and PSS.

structure. The reaction of the PEDOT and the PSS was shown in Figure 2.

The conductivity of the PEDOT: PSS was enhanced through adding the solvent, such as sorbitol. The sorbitol treatment screens the ionic interaction between PEDOT and PSS to form ionic bonding. The screening effect led to better phase separation between PEDOT and PSS. Thus, the coiled type of PEDOT and PSS chains was reoriented to the linear type of PEDOT and PSS chains [18-20]. The schematic illustration of the reorientation of PEDOT: PSS was shown in Figure 3. In addition, additives of the polyol caused PEDOT chains aggregation $[16,21,22]$.

The conductivity of the PEDOT: PSS was further enhanced through adding the dilute sulfuric acid again. Figure 4 shows that the sheet resistance and surface roughness of the doped PEDOT: PSS film through adding $1.5 \mathrm{M} \mathrm{H}_{2} \mathrm{SO}_{4}$ was measured by four-point probe and AFM, 


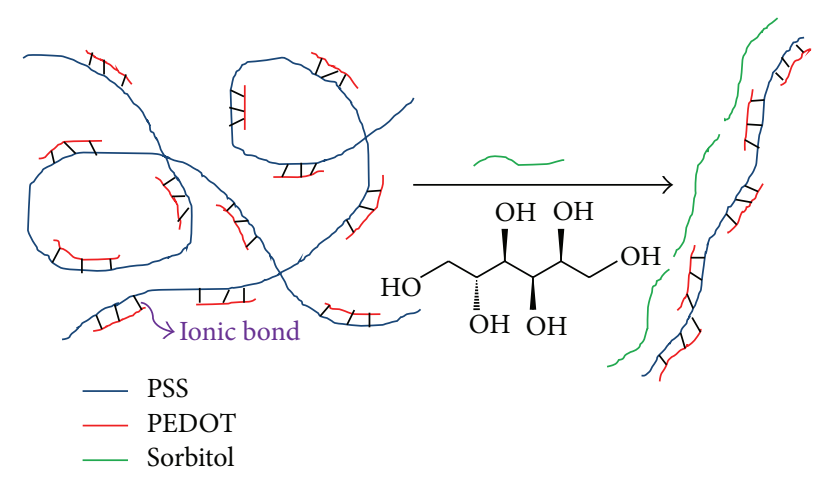

FIGURE 3: Schematic illustration of the reorientation of PEDOT: PSS by sorbitol.

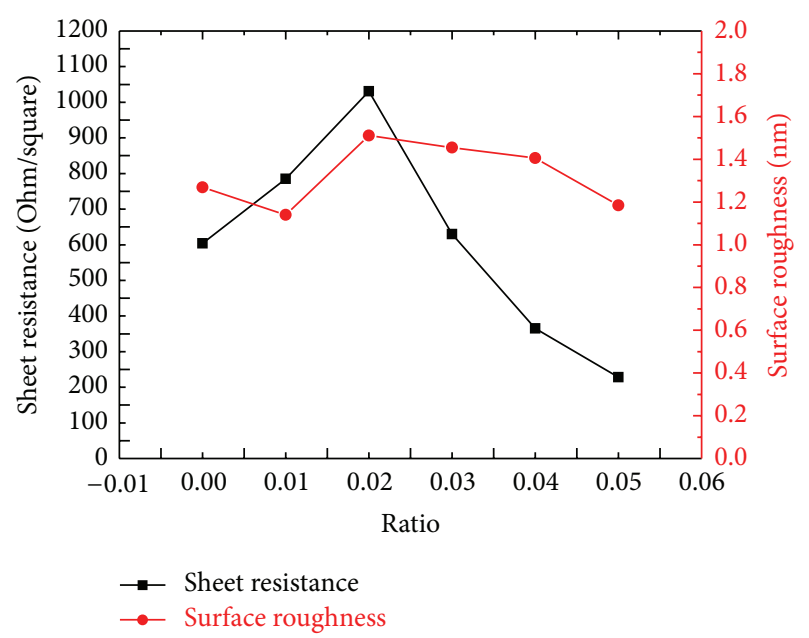

FIGURE 4: The sheet resistance and surface roughness of doped PEDOT: PSS with different weight ratios of $1.5 \mathrm{M} \mathrm{H}_{2} \mathrm{SO}_{4}$ to PEDOT: PSS.

respectively. It is clear that the sheet resistance of the doped PEDOT: PSS film is affected by adding the various ratios of $\mathrm{H}_{2} \mathrm{SO}_{4}$. The sheet resistance of the doped PEDOT: PSS film enhanced with increasing the ratio of $\mathrm{H}_{2} \mathrm{SO}_{4}$, but it drops after the maximum sheet resistance. The value of sheet resistance is reduced from 604 to $228 \Omega / \mathrm{sq}$. The surface roughness of the double doped PEDOT: PSS film is not almost changed.

Figure 5 shows the sheet resistance and surface roughness of the doped PEDOT: PSS film through adding $1 \mathrm{M} \mathrm{H}_{2} \mathrm{SO}_{4}$. The sheet resistance value is reduced from 604 to $255 \Omega / \mathrm{sq}$. The surface roughness of the $1 \mathrm{M} \mathrm{H}_{2} \mathrm{SO}_{4}$ doped PEDOT: PSS film was smoother as compared with the $1.5 \mathrm{M} \mathrm{H}_{2} \mathrm{SO}_{4}$ doped PEDOT: PSS film. Although the sheet resistance and surface roughness were improved slightly, we cannot clearly find the tendency from the $1 \mathrm{M}$ and $1.5 \mathrm{M} \mathrm{H}_{2} \mathrm{SO}_{4}$ doped PEDOT: PSS films. This is due to the fact that the reaction rate of high concentration of $\mathrm{H}_{2} \mathrm{SO}_{4}$ is too fast. Thus, we reduce the molar concentration of $\mathrm{H}_{2} \mathrm{SO}_{4}$ again.

The reaction rate of $\mathrm{H}_{2} \mathrm{SO}_{4}$ was slowed down when the molar concentration of $\mathrm{H}_{2} \mathrm{SO}_{4}$ was $0.5 \mathrm{M}$. The results are shown in Figure 6 . The sheet resistance and surface roughness

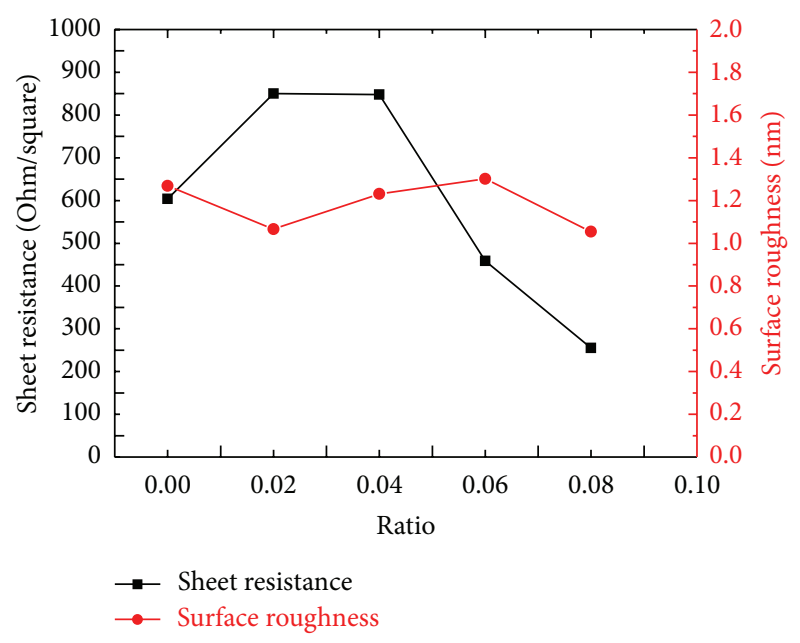

FIGURE 5: The sheet resistance and surface roughness of doped PEDOT: PSS with different weight ratios of $1 \mathrm{M} \mathrm{H}_{2} \mathrm{SO}_{4}$ to PEDOT: PSS.

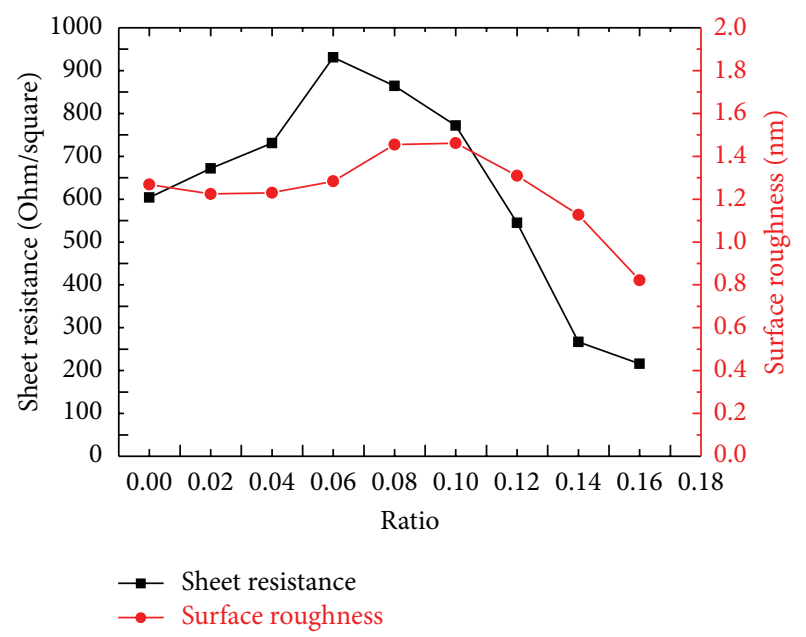

FIGURE 6: The sheet resistance and surface roughness of doped PEDOT: PSS with different weight ratios of $0.5 \mathrm{M} \mathrm{H}_{2} \mathrm{SO}_{4}$ to PEDOT: PSS.

were reduced significantly. The sheet resistance is reduced from 604 to $216 \Omega / \mathrm{sq}$ and the surface roughness is also reduced from 1.268 to $0.822 \mathrm{~nm}$. Furthermore, we further decreased again the molar concentration of $\mathrm{H}_{2} \mathrm{SO}_{4}$. However, the PEDOT: PSS solution was diluted with the lower molar concentration of $\mathrm{H}_{2} \mathrm{SO}_{4}$.

The various molar concentrations of $\mathrm{H}_{2} \mathrm{SO}_{4}$ were added to the PEDOT: PSS. It can be seen that there is a common phenomenon. The sheet resistance was slightly increased with adding a small amount of $\mathrm{H}_{2} \mathrm{SO}_{4}$. The reason for this phenomenon is that the sulfuric acid was reacted with sorbitol preferentially. The reaction is called the pinacol rearrangement. It resulted that the reorient and aggregation effect combined PEDOT grains with sorbitol was destroyed. After the reaction of the pinacol rearrangement, the residual $\mathrm{H}_{2} \mathrm{SO}_{4}$ reacted with PSS. The chemical reaction can be written as $\mathrm{H}_{2} \mathrm{SO}_{4}+\mathrm{PSS}^{-} \rightarrow \mathrm{HSO}_{4}{ }^{-}+\mathrm{PSSH}$. The $\mathrm{HSO}_{4}{ }^{-}$ 


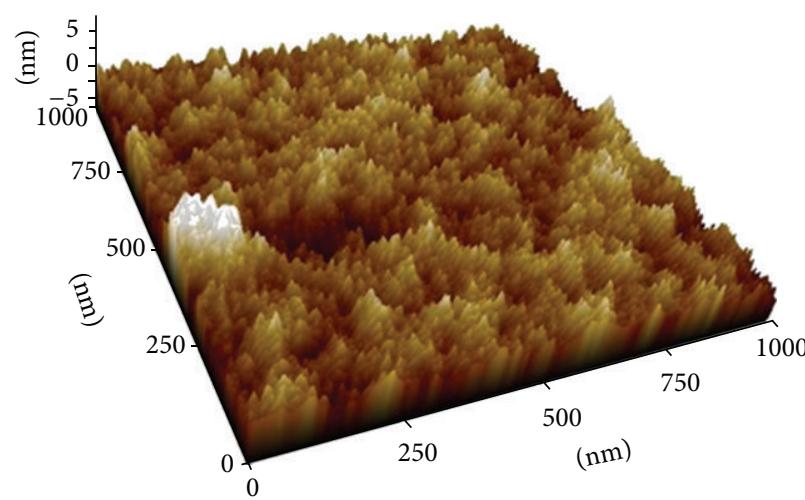

(a)

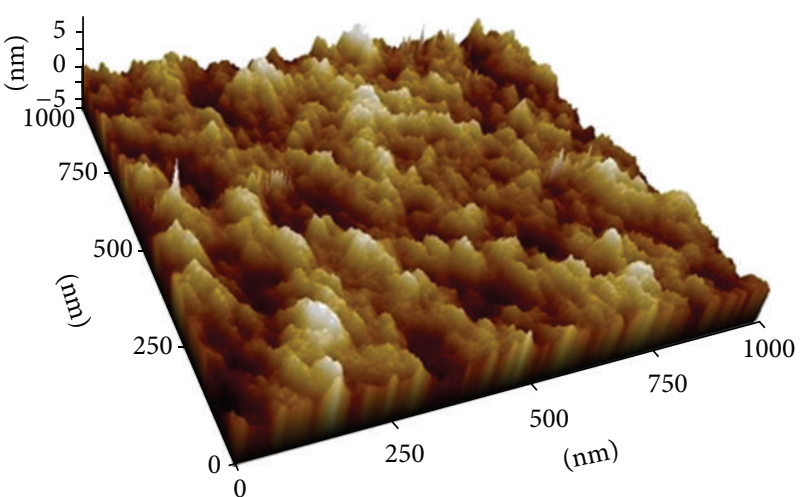

(b)

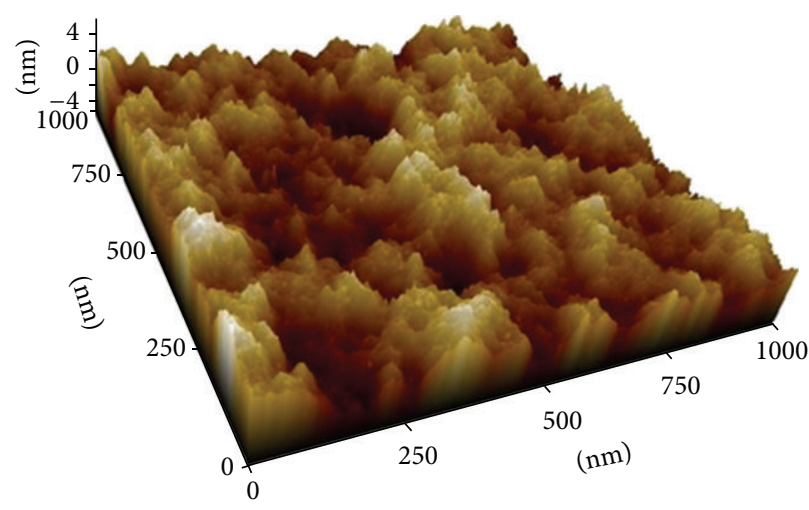

(c)

FIgURE 7: The surface morphology of (a) pristine PEDOT: PSS; (b) doping ratio of PEDOT: PSS to dilute $\mathrm{H}_{2} \mathrm{SO}_{4}$ is $25: 2$; (c) doping ratio of PEDOT: PSS to dilute $\mathrm{H}_{2} \mathrm{SO}_{4}$ is $25: 4$.

formed an ionic bond with the PEDOT, even that the nonconductive anions of some PSS ${ }^{-}$were substituted by the conductive anions of $\mathrm{HSO}_{4}^{-}$[23]. The ionic bond reaction is similar to Figure 2. And the substitution reaction caused more aggregation of PEDOT chains. The results are shown in Figure 7. The higher phase signal in Figure 7 corresponds to the PEDOT, and the lower phase was the PSS [15, 22, 24]. It's clear that PEDOT chains were increasingly aggregated with increasing the ratio of $\mathrm{H}_{2} \mathrm{SO}_{4}$. Thus, the substitution reaction will be favorable for the conductivity enhancement.

Figure 8 shows the doped PEDOT: PSS films with different molar $\mathrm{H}_{2} \mathrm{SO}_{4}$ concentrations as a function of weight ratio of $\mathrm{H}_{2} \mathrm{SO}_{4}$ to PEDOT: PSS. The transparency of the doped PEDOT: PSS film can be affected by the $\mathrm{H}_{2} \mathrm{SO}_{4}$ treatment. The transmittance of the doped PEDOT: PSS film was decreased with increasing the ratio of $\mathrm{H}_{2} \mathrm{SO}_{4}$ to PEDOT: PSS. The lowest sheet resistance of $216 \Omega / \mathrm{sq}$ is obtained at $0.5 \mathrm{M} \mathrm{H}_{2} \mathrm{SO}_{4}$-doped PEDOT: PSS film and at the weight ratio of 0.16 whose transmittance in the visible wavelength range from 400 to $700 \mathrm{~nm}$ is $91.9 \%$. The high transparency and low sheet resistance indicated that the $0.5 \mathrm{M} \mathrm{H}_{2} \mathrm{SO}_{4}$-doped PEDOT: PSS films can be used as the transparent conductive electrode of optoelectronic devices.

\section{Conclusions}

In this paper, it was employed the noncontact AFM, four point sheet resistivity meter, and U-3900 spectroscopy to investigate the origin of the sheet resistance decrease of $\mathrm{H}_{2} \mathrm{SO}_{4}$-doped PEDOT: PSS films. The doped PEDOT: PSS solution by adding the different molar concentrations of $\mathrm{H}_{2} \mathrm{SO}_{4}$ strongly affects the surface roughness, sheet resistance, and transmittance. After doped $\mathrm{H}_{2} \mathrm{SO}_{4}$, the surface roughness is reduced from $1.268 \mathrm{~nm}$ to $0.822 \mathrm{~nm}$. It's indicated that the surface of $\mathrm{H}_{2} \mathrm{SO}_{4}$-doped PEDOT: PSS film was smoother compared with the doped PEDOT: PSS film. The pinacol rearrangement occurred by adding the dilute sulfuric acid, but the sheet resistance was improved from 604 to $216 \Omega /$ sq. The decrease of the sheet resistance is due to the fact that the nonconductive anions of some PSS $^{-}$were substituted by the conductive anions of $\mathrm{HSO}_{4}{ }^{-}$. And the substitution reaction caused more aggregation of PEDOT chains; namely, the substitution reactions will be favorable for the conductivity enhancement. The transmittance of the doped PEDOT: PSS film is decreased with increasing the weight ratio of $\mathrm{H}_{2} \mathrm{SO}_{4}$ to PEDOT: PSS. The transmittance of the $0.5 \mathrm{M} \mathrm{H}_{2} \mathrm{SO}_{4}$-doped PEDOT: PSS film is above $91 \%$ in the visible wavelength range from 400 to $700 \mathrm{~nm}$. However, the high transparency and low sheet resistance reveal that 


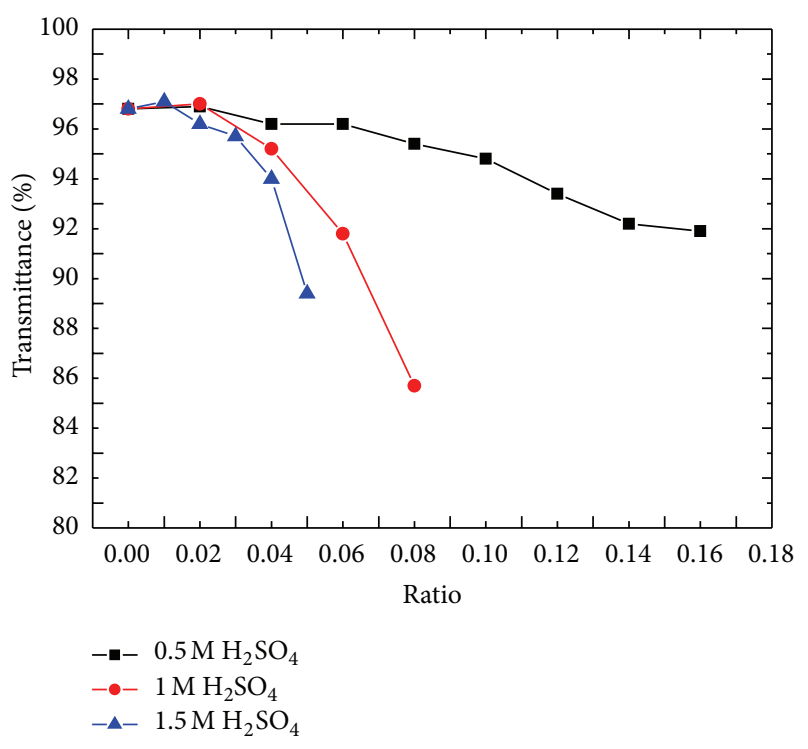

FIGURE 8: The transmittance of the doped PEDOT: PSS with different molar concentrations of $\mathrm{H}_{2} \mathrm{SO}_{4}$ as a function of weight ratio of $\mathrm{H}_{2} \mathrm{SO}_{4}$ to PEDOT: PSS.

the $\mathrm{H}_{2} \mathrm{SO}_{4}$-doped PEDOT: PSS films can be used as the transparent conductive electrode of optoelectronic devices.

\section{Conflict of Interests}

The authors declare that there is no conflict of interests regarding the publication of this paper.

\section{Acknowledgments}

This work was partially supported by the National Science Council of Taiwan under Contract no. NSC102-2221-E-390019-MY2.

\section{References}

[1] S. Park, S. J. Tark, and D. Kim, "Effect of sorbitol doping in PEDOT:PSS on the electrical performance of organic photovoltaic devices," Current Applied Physics, vol. 11, no. 6, pp. 12991301, 2011.

[2] B. Zhang, W. Li, J. Yang et al., "Performance enhancement of polymer light-emitting diodes by using ultrathin fluorinated polyimide modifying the surface of poly(3,4-ethylene dioxythiophene):poly(styrenesulfonate)," Journal of Physical Chemistry C, vol. 113, no. 18, pp. 7898-7903, 2009.

[3] G. Latessa, F. Brunetti, A. Reale, G. Saggio, and A. Di Carlo, "Piezoresistive behaviour of flexible PEDOT:PSS based sensors," Sensors and Actuators B, vol. 139, no. 2, pp. 304-309, 2009.

[4] A. Onorato, M. A. Invernale, I. D. Berghorn, C. Pavlik, G. A. Sotzing, and M. B. Smith, "Enhanced conductivity in sorbitoltreated PEDOT-PSS. Observation of an in situ cyclodehydration reaction," Synthetic Metals, vol. 160, no. 21-22, pp. 22842289, 2010.
[5] A. K. Havare, M. Can, S. Demic, M. Kus, and S. Icli, "The performance of OLEDs based on sorbitol doped PEDOT:PSS," Synthetic Metals, vol. 161, no. 23-24, pp. 2734-2738, 2012.

[6] A. M. Nardes, M. Kemerink, M. M. de Kok, E. Vinken, K. Maturova, and R. A. J. Janssen, "Conductivity, work function, and environmental stability of PEDOT:PSS thin films treated with sorbitol," Organic Electronics, vol. 9, no. 5, pp. 727-734, 2008.

[7] C. J. Huang, K. L. Chen, Y. J. Tsao, D.-W. Chouc, W.-R. Chend, and T.-H. Meend, "Study of solvent-doped PEDOT: PSS layer on small molecule organic solar cells," Synthetic Metals, vol. 164, pp. 38-41, 2013.

[8] J.-H. Huang, D. Kekuda, C.-W. Chu, and K.-C. Ho, "Electrochemical characterization of the solvent-enhanced conductivity of poly(3,4-ethylenedioxythiophene) and its application in polymer solar cells," Journal of Materials Chemistry, vol. 19, no. 22, pp. 3704-3712, 2009.

[9] I. Cruz-Cruz, M. Reyes-Reyes, M. A. Aguilar-Frutis, A. G. Rodriguez, and R. López-Sandoval, "Study of the effect of DMSO concentration on the thickness of the PSS insulating barrier in PEDOT:PSS thin films," Synthetic Metals, vol. 160, no. 13-14, pp. 1501-1506, 2010.

[10] J. Luo, D. Billep, T. Waechtler et al., "Enhancement of the thermoelectric properties of PEDOT:PSS thin films by posttreatment," Journal of Materials Chemistry A, vol. 1, no. 26, pp. 7576-7583, 2013.

[11] M. S. Kim, S. K. Park, Y.-H. Kim, J. W. Kang, and J.-I. Han, "Glycerol-doped poly(3,4-ethylenedioxy-thiophene): Poly (styrene sulfonate) buffer layer for improved power conversion in organic photovoltaic devices," Journal of the Electrochemical Society, vol. 156, no. 10, pp. H782-H785, 2009.

[12] M.-W. Lee, M.-Y. Lee, J.-C. Choi, J.-S. Park, and C.-K. Song, "Fine patterning of glycerol-doped PEDOT:PSS on hydrophobic PVP dielectric with ink jet for source and drain electrode of OTFTs," Organic Electronics, vol. 11, no. 5, pp. 854-859, 2010.

[13] K. H. Tsai, S. C. Shiu, and C. F. Lin, "Improving the conductivity of hole injection layer by heating PEDOT:PSS," in Organic Photovoltaics IX, vol. 7052 of Proceedings of SPIE, no. 1, 2008.

[14] Z. Hu, J. Zhang, Z. Hao, and Y. Zhao, "Influence of doped PEDOT:PSS on the performance of polymer solar cells," Solar Energy Materials and Solar Cells, vol. 95, no. 10, pp. 2763-2767, 2011.

[15] X. Crispin, F. L. E. Jakobsson, A. Crispin et al., "The origin of the high conductivity of poly(3,4-ethylenedioxythiophene)-poly (styrenesulfonate) (PEDOT-PSS) plastic electrodes," Chemistry of Materials, vol. 18, no. 18, pp. 4354-4360, 2006.

[16] H. Yan and H. Okuzaki, "Effect of solvent on PEDOT/PSS nanometer-scaled thin films: XPS and STEM/AFM studies," Synthetic Metals, vol. 159, no. 21-22, pp. 2225-2228, 2009.

[17] D. A. Mengistie, P. C. Wangc, and C. W. Chu, "Effect of molecular weight of additives on the conductivity of PEDOT: PSS and efficiency for ITO-free organic solar cells," Journal of Materials Chemistry A, vol. 1, no. 34, pp. 9907-9915, 2013.

[18] J. Y. Kim, J. H. Jung, D. E. Lee, and J. Joo, "Enhancement of electrical conductivity of poly(3,4-ethylenedioxythiophene)/poly (4-styrenesulfonate) by a change of solvents," Synthetic Metals, vol. 126, no. 2-3, pp. 311-316, 2002.

[19] L. A. A. Pettersson, S. Ghosh, and O. Inganäs, "Optical anisotropy in thin films of poly(3,4-ethylenedioxythiophene)poly(4-styrenesulfonate)," Organic Electronics, vol. 3, no. 3-4, pp. 143-148, 2002. 
[20] S. Timpanaro, M. Kemerink, F. J. Touwslager, M. M. De Kok, and S. Schrader, "Morphology and conductivity of PEDOT/PSS films studied by scanning-tunneling microscopy," Chemical Physics Letters, vol. 394, no. 4-6, pp. 339-343, 2004.

[21] J. Ouyang, Q. Xu, C.-W. Chu, Y. Yang, G. Li, and J. Shinar, "On the mechanism of conductivity enhancement in poly $(3,4$ ethylenedioxythiophene):poly(styrene sulfonate) film through solvent treatment," Polymer, vol. 45, no. 25, pp. 8443-8450, 2004.

[22] Y.-S. Hsiao, W.-T. Whang, C.-P. Chen, and Y.-C. Chen, "Highconductivity poly(3,4-ethylenedioxythiophene):poly(styrene sulfonate) film for use in ITO-free polymer solar cells," Journal of Materials Chemistry, vol. 18, no. 48, pp. 5948-5955, 2008.

[23] Y. Xia, K. Sun, and J. Ouyang, "Solution-processed metallic conducting polymer films as transparent electrode of optoelectronic devices," Advanced Materials, vol. 24, no. 18, pp. 24362440, 2012.

[24] Y. Wang, R. Song, Y. Li, and J. Shen, "Understanding tappingmode atomic force microscopy data on the surface of soft block copolymers," Surface Science, vol. 530, no. 3, pp. 136-148, 2003. 

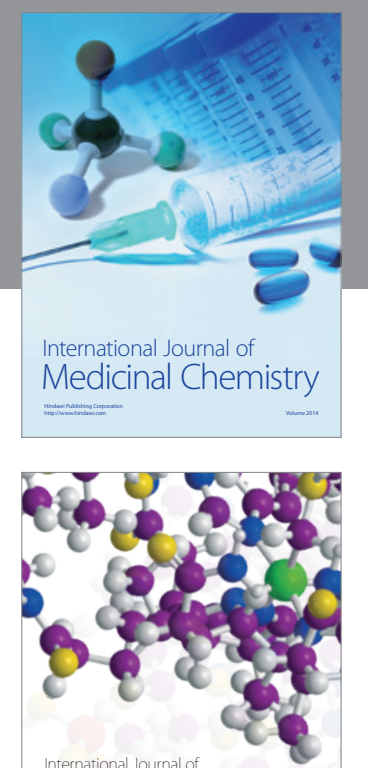

\section{Carbohydrate} Chemistry

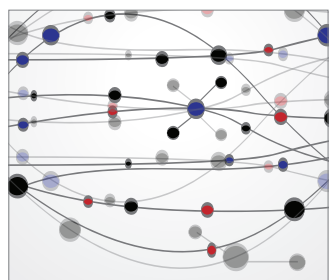

The Scientific World Journal
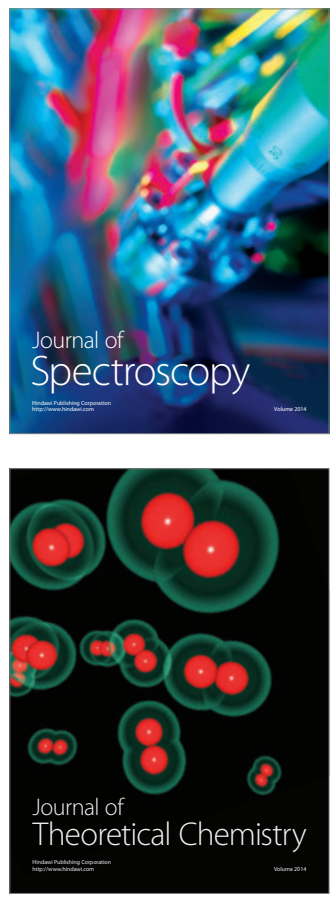
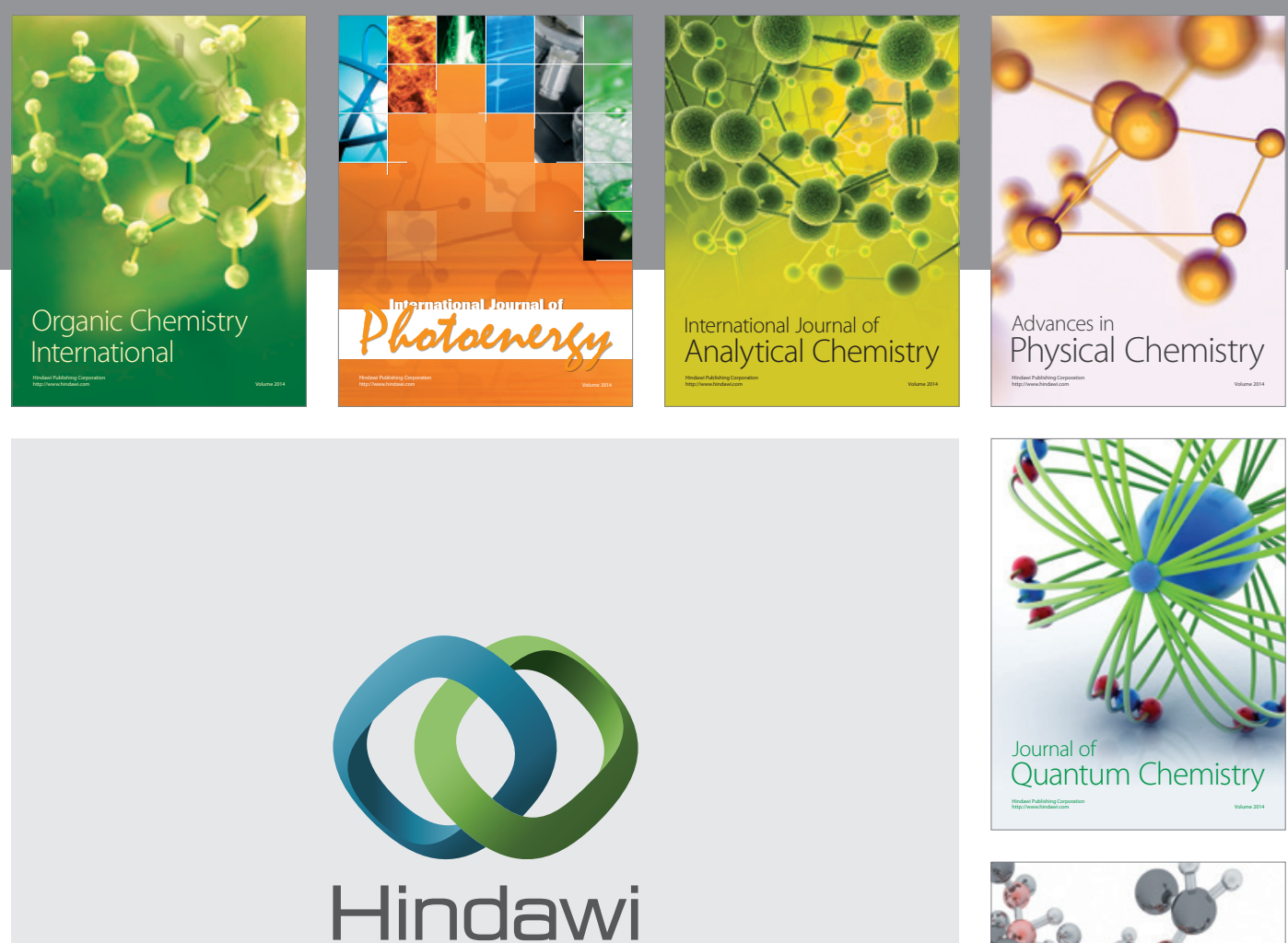

Submit your manuscripts at

http://www.hindawi.com

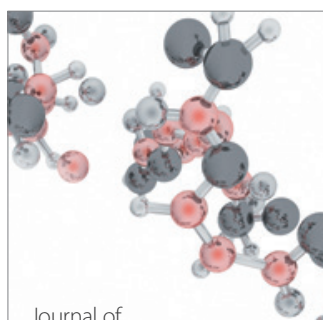

Analytical Methods

in Chemistry

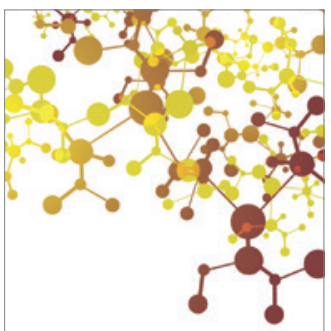

Journal of

Applied Chemistry

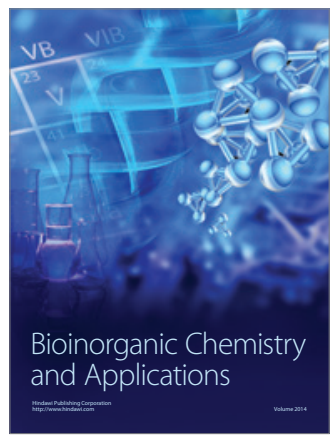

Inorganic Chemistry
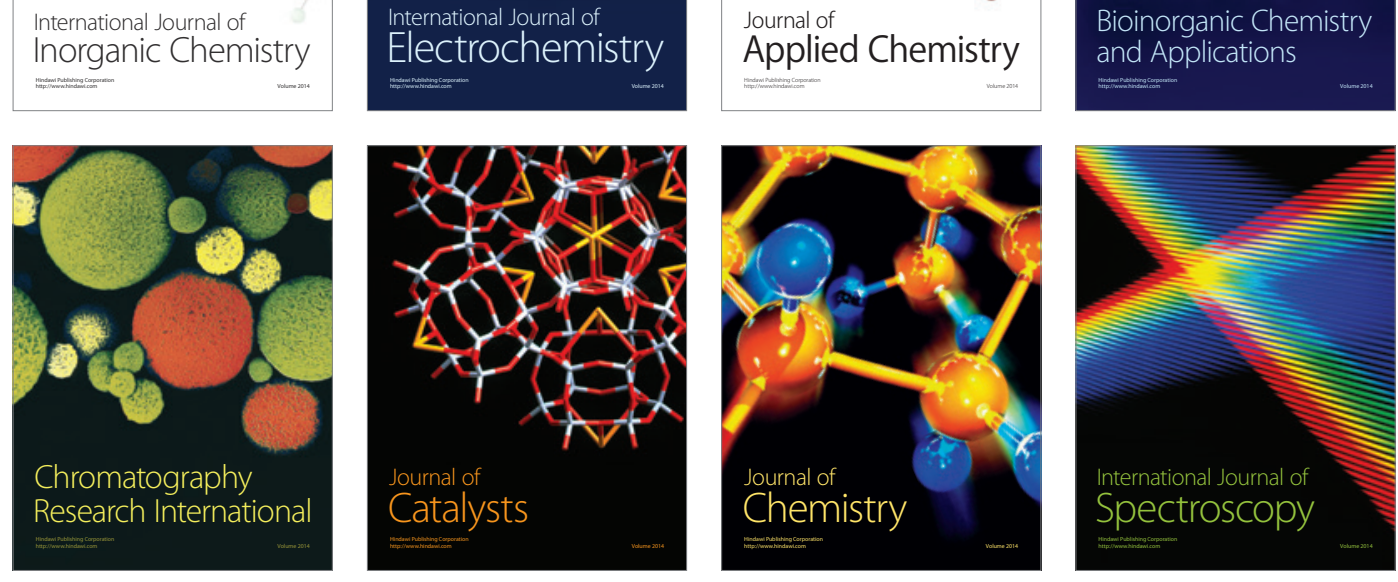